\title{
Descrição do perfil nutricional e alimentar dos funcionórios de um serviço público
}

\section{Description of the nutritional and eating profile of the workers of a public service}

\author{
Camila Graciolal; Fernanda Vaz Fortuna ${ }^{2}$ \\ ${ }^{1}$ Graduada em Nutrição - Univates.Lajeado, RS [Brasil] \\ ${ }^{2}$ Especialista em Nutrição Clínica, Docente do curso de Nutrição - Univates.Lajeado, RS [Brasil] \\ Endereço para correspondência \\ Camila Graciola \\ RS 129 KM 72, 6040 \\ 95960-000 - Encantado, RS [Brasil] \\ milagraciola@ig.com.br
}

\begin{abstract}
Resumo
Objetivos: Utilizaram-se o IMC e a CC para medir o estado nutricional, e o QFA e o Guia Alimentar, para determinar as porções do consumo diário de alimentos. Método: Neste estudo transversal, os dados categóricos foram descritos em percentuais, e os quantitativos, em média e desvio-padrão. A significância foi determinada pelo teste " $\mathrm{t}$ " Student. A amostra final foi composta por 68 participantes. Resultados: O IMC revelou 55,88\%, com eutrofia; 30,88\%, com sobrepeso, e $13,23 \%$ com obesidade. A CC mostrou risco aumentado em $17,65 \%$, e muito aumentado, em $17,65 \%$. Em relação ao consumo alimentar, as médias de porções consumidas foram: $4,94 \pm 1,24$, cereais; $0,34 \pm 0,68$, leguminosas; $1,62 \pm 0,88$, carnes; $1,62 \pm 1,07$, leite; $1,28 \pm 1,22$, hortaliças; $1,53 \pm 1,62$, frutas e $1,97 \pm 1,26$, açucares, e 1,72 $\pm 1,02$, gorduras. Conclusões: $\mathrm{O}$ aumento do excesso de peso e a alimentação inadequada da população mostram a necessidade de ações que visem à prevenção e ao controle de doenças, contribuindo para a redução de enfermidades crônicas relacionadas a ambos os fatores.
\end{abstract}

Descritores: Estado Nutricional; Obesidade; Consumo de alimentos.

\begin{abstract}
Objectives: The aim of this work was to describe the nutritional and eating profile of the workers of a public service. Method: The Body Mass Index (BMI) was used and also the circumference of the waist (WC). A transversal delimitation, categorical data described as percentile and quantitative in average and standard deviation. The significance was determined through Student's t- test. The final sample was composed by 68 participants. Results: The BMI revealed 55,88\% with eutrophia, followed by overweight in $30,88 \%$ and obesity in $13,23 \%$. The waist circumference revealed increased risk in $17,65 \%$ and very increased risk in another $17,65 \%$. Regarding food intake, the average portions consumed were $4,94 \pm 1,22$; legumes $0,34 \pm 0,68$; meats $1,62 \pm 0,88$; dairy $1,62 \pm 1,07$; vegetables 1,28 $\pm 1,22$; fruit $1,53 \pm 1,62$ and sugars $1,97 \pm 1,26$; and fats $1,72 \pm 1,02$. Conclusions: The increase of weight excess and inadequate feeding habits of population show the necessity of actions aimed at the prevention and control of diseases, contributing to the reduction of chronic illness related to this both factors.
\end{abstract}

Key words: Nutritional status; Obesity; Food consumption. 


\section{Introdução}

O aumento da prevalência de obesidade observado nas últimas décadas tem sido atribuído, principalmente, às mudanças nos hábitos alimentares da população, com maior consumo de alimentos de alta densidade energética e industrializados; inatividade física, e a outros fatores de caráter sociocultural, por exemplo, a valorização do excesso de peso como sinônimo de saúde e prosperidade ${ }^{1,2,3}$. Essas são tendências de transição nutricional decorrentes da urbanização e industrialização ocorridas neste século, direcionando para uma dieta mais ocidentaliza$\mathrm{da}$, com alimentos ricos em calorias, em especial destaque para o maior consumo de carnes, leite e derivados ricos em lipídios, redução do consumo de frutas, cereais, verduras e legumes ${ }^{4,5,6,7}$.

A obesidade é um problema de saúde pública de todos os países. Especialistas em nutrição e dietética e as autoridades de saúde destacam a importância da relação entre dieta e saúde; preconizando uma alimentação com menos calorias, lipídios e sacarose juntamente com a realização de atividade física ${ }^{8}$. A preocupação desses profissionais é decorrente de seu entendimento de que a obesidade, não é apenas o resultado de pessoas que exageram no consumo de alimentos, ou uma questão estética, mas que pode estar relacionada a comorbidades e doenças crônicas, tais como diabetes mellitus (DM), hipertensão e doenças cardiovasculares ${ }^{3,}$ 8,9,10. As evidências científicas também mostram que alimentos de origem vegetal, principalmente frutas, legumes e verduras, se consumidos de forma regular e em quantidades apropriadas, são fatores de proteção contra várias doenças relacionadas à alimentação, contribuindo para a manutenção de um peso saudável ${ }^{11}$.

$\mathrm{O}$ acúmulo do excesso de tecido adiposo corporal tem sido tradicionalmente diagnosticado pelo indicador antropométrico Índice de Massa Corporal (IMC) ${ }^{12}$, o qual apresenta relação com a massa adiposa e com as alterações metabólicas associadas à obesidade ${ }^{13}$. E a circunferência da cintura, que define a distribuição de tecido adiposo abdominal, é relacionada como importante fator de risco para as doenças crônicas não transmissíveis (DCNT) ${ }^{13}$.

$\mathrm{O}$ aumento da tendência a excesso de peso e obesidade na população brasileira comprova que o problema está assumindo graves proporções, o que fundamenta a necessidade de intervenções que façam retroceder esse avanço e, concomitantemente, de outras DCNTs. A implementação de programas que possam melhorar a situação econômica deve ser desenvolvida para que a população tenha hábitos de vida adequados $^{10}$. Este artigo tem como objetivo descrever o perfil nutricional e alimentar dos funcionários de um serviço público do interior do Rio Grande do Sul (RS).

\section{Materiais e métodos}

Foi realizado um estudo transversal, constituído por funcionários de um serviço público numa cidade no interior do Rio Grande do Sul. A coleta dos dados foi realizada em sala privativa com atendimento individualizado, no mês de julho de 2009. A amostra final foi composta por 68 participantes. Os critérios de exclusão foram: indivíduos com idade superior a 60 anos ou inferior a 20 anos, portadores de necessidades especiais e gestantes.

Foram estudadas as seguintes variáveis: idade, em anos completos; gênero; renda mensal, distribuída por faixas de um a dois, dois a cinco e mais do que cinco salários mínimos, (de acordo com um salário mínimo vigente em agosto/2009, no valor de R 465,00 ); tabagismo, sendo considerados fumantes aqueles que fumavam qualquer quantidade de cigarro diariamente; atividade física, foi classificado como praticante quem executava qualquer frequência de atividade entre as opções: todos os dias, uma vez por semana e três a seis vezes por semana, e com nenhuma frequência, como não praticante de atividade física; frequência de consumo de bebidas alcoólicas, considerou-se usuário os que faziam uso diário, eventual, apenas fins de 
semana e não utilização, aqueles que não as ingeriam; patologias apresentadas, história de doença familiar, uso de medicamentos e orientação alimentar referente a patologia. Essas informações foram obtidas por meio de um questionário de autopreenchimento com perguntas fechadas.

O questionário de pesquisa foi aprovado pelo Comitê de Ética em Pesquisas do Centro Universitário Univates, sob o número 054/09, conforme as normas estabelecidas na Resolução 196/96, do Conselho Nacional de Saúde para estudos envolvendo seres humanos. Após detalhado e esclarecido os propósitos desta investigação, os procedimentos utilizados, os benefícios e os possíveis riscos atrelados, os sujeitos assinaram o Termo de Consentimento Livre e Esclarecido, condicionando sua participação de modo voluntário.

O estado nutricional foi identificado pelas medidas de peso, altura, IMC e circunferência da cintura (CC). Para a aferição dessas medidas antropométricas, os participantes se apresentavam descalços, usando roupas leves e sem chapéu ou adornos na cabeça, seguindo as recomendações do Manual de Técnicas e Procedimentos ${ }^{14}$. Os indivíduos mantiveram-se em pé de costas para a balança, descalços, com os calcanhares juntos, costas retas e os braços estendidos ao lado do corpo. O peso foi registrado em quilograma, obtido por meio de balanças da marca Plenna ${ }^{\circledR}$ Litthium Digital, modelo MEA - 08128, do tipo portátil com capacidade para $150 \mathrm{~kg}$ e variação de $0,1 \mathrm{~kg}$. A estatura foi aferida utilizando-se um estadiômetro portátil Seca ${ }^{\circledR}$, modelo Body Metter 208. Para medir a altura, foi solicitado ao indivíduo que permanecesse olhando para um ponto fixo na altura dos olhos e em movimento de inspiração ${ }^{14}$.

A medida da CC foi obtida ao nível da cintura natural (parte mais estreita do abdome) sem fazer pressão com o auxílio de uma fita métrica inextensível de material resistente, marca Cardiomed ${ }^{\circledR}$ com precisão de $0,1 \mathrm{~cm}$, estando o indivíduo despido nessa região, em pé, com os pés juntos, os braços estendidos lateralmente e o abdome relaxado. A leitura foi feita no centíme- tro mais próximo em que a fita cruzava o ponto zero, entre uma expiração e uma inspiração. Para a classificação dessa medida, foram utilizados os pontos de corte recomendados para avaliar o risco de complicações metabólicas, com os quais se considera risco aumentado para homens os valores de CC maior ou igual a $94 \mathrm{~cm}$, e substancialmente aumentados, os iguais ou superiores a $102 \mathrm{~cm}$; e para as mulheres, risco aumentado se a CC for maior ou igual a $80 \mathrm{~cm}$, e muito aumentado, para valores iguais ou superiores a 88 $\mathrm{cm}$, conforme a Organização Mundial da Saúde $(\mathrm{OMS})^{15}$.

O IMC foi obtido pelo quociente da massa corporal e estatura ${ }^{2}$, cujo valor foi expresso em $\mathrm{kg} / \mathrm{m}^{2}$, conforme determinações do Ministério da Saúde ${ }^{14}$. Os pontos de corte selecionados para a classificação do IMC foram: normalidade (18,5$\left.24,9 \mathrm{~kg} / \mathrm{m}^{2}\right)$, sobrepeso $\left(25,0-29,9 \mathrm{~kg} / \mathrm{m}^{2}\right)$ e obesidade geral $\left(\geq 30,0 \mathrm{~kg} / \mathrm{m}^{2}\right)$, conforme estabelecido pela $\mathrm{OMS}^{15}$.

A qualidade da alimentação foi identificada pelo questionário validado de frequência alimentar (QFA) ${ }^{16}$. O instrumento é composto por 52 itens alimentares, ordenados em nove grupos: cereais, leguminosas, óleos, sobremesas e doces, petiscos e enlatados, carnes e ovos, leites e derivados, hortaliças e frutas, bebidas, produtos diet e light. No grupo das hortaliças e frutas, foram obtidos os dois representantes mais frequentemente consumidos. As respostas foram transformadas em porções de consumo diário e comparadas às recomendações do Guia Alimentar para população brasileira. Para comparar tais respostas com o preconizado nesse guia foi considerado o consumo diário: uma vez por dia e cinco a seis vezes por semana.

Foi utilizada a tabela das medidas caseiras do Guia Alimentar Brasileiro, para obter as porções consumidas de cada grupo alimentar ${ }^{11}$. $\mathrm{Na}$ análise dos resultados, não foram considerados os grupos: diet e light, petiscos e enlatados, pois nesta pesquisa o consumo encontrado desses alimentos foi muito pequeno e tornou-se insuficiente para comparar a porção consumida diariamente com o aconselhado no guia. 
O número de porções encontrado no estudo foi comparado com o de porções proposto, abaixo do proposto e acima do proposto em relação às Diretrizes Alimentares Brasileiras ${ }^{11}$. Foram analisados oito grupos alimentares: cereais, leguminosas, carnes, leites e derivados, hortaliças, frutas, açucares e gorduras ${ }^{11}$.

A análise estatística foi realizada com o auxílio do software Bioestat 5.0 versão $2007^{17}$. Os dados categóricos foram descritos em percentuais e os quantitativos em média e desvio-padrão $(\mathrm{m} \pm \mathrm{dp})$. A significância estatística foi determinada pelo teste " $\mathrm{t}$ " Student, ou Teste G, considerando significante um $p<0,05$. A força de correlação foi estabelecida pela correlação de Pearson ou teste de Matriz de correlação.

\section{Resultados}

A amostra pesquisada totalizou 68 participantes, sendo a perda igual a $0,12 \%$; a maior parte dos funcionários era do sexo feminino, correspondendo a $42(61,8 \%)$ mulheres. As características demográficas e antropométricas são descritas na Tabela 1.
Em relação ao IMC, a maioria dos pesquisados foi classificada no estado nutricional de eutrofia (38 indivíduos ou 55,88\% da amostra) e o restante por sobrepeso (21 voluntários ou $30,88 \%$ ), e obesidade (9 participantes ou 13,23\%). A média do IMC geral encontrada foi $28 \pm 3,85 \mathrm{Kg} /$ $\mathrm{m}^{2}$. A faixa etária variou entre 20 e 60 anos, sendo a idade média $36 \pm 11,89$ anos. Podemos analisar na Tabela 2 a relação do estado nutricional com alguns fatores de risco, havendo diferença significativa nos seguintes variáveis: idade, renda, CC feminina e CC masculina, conforme a classificação do IMC.

Tabela 1: Características demográficas e antropométricas dos funcionários de um serviço público do interior do RS

\begin{tabular}{c|c|c|c}
\hline $\begin{array}{c}\text { Variável/ } \\
\text { sexo }\end{array}$ & $\begin{array}{c}\text { Feminino } \\
(\mathrm{m} \pm \mathrm{dp})\end{array}$ & $\begin{array}{c}\text { Masculino } \\
(\mathrm{m} \pm \mathrm{dp})\end{array}$ & $\mathrm{p}^{*}$ \\
\hline Idade (anos) & $36,74 \pm 11,35$ & $36,42 \pm 12,95$ & 0,916 \\
\hline $\mathrm{CC}(\mathrm{cm})$ & $78,36 \pm 10,89$ & $88,19 \pm 11,34$ & 0,001 \\
\hline $\mathrm{IMC}\left(\mathrm{kg} / \mathrm{m}^{2}\right)$ & $24,73 \pm 3,86$ & $26,06 \pm 3,74$ & 0,166 \\
\hline Peso $(\mathrm{kg})$ & $65,45 \pm 11,11$ & $78,42 \pm 11,52$ & $<0,001$ \\
\hline
\end{tabular}

CC: circunferência da cintura. IMC: índice de massa corporal. Valores expressos como média $(\mathrm{m}) \pm$ desvio-padrão $(\mathrm{dp})$.* Teste " $\mathrm{t}$ " para amostras independentes $p$ significante quando $<0,05$.

Tabela 2: Fatores associados ao estado nutricional em funcionários de um serviço público do interior do RS

\begin{tabular}{|c|c|c|c|c|}
\hline Fatores de risco & Eutrofia n (\%) & Sobrepeso n (\%) & Obesidade n (\%) & $P^{*}$ \\
\hline $\begin{array}{l}\text { Idade (anos) } \\
20-29 \\
30-39 \\
40-49 \\
50-59\end{array}$ & $\begin{array}{l}19(27,94 \%) \\
10(14,70 \%) \\
6(8,82 \%) \\
4(5,88 \%)\end{array}$ & $\begin{array}{c}5(7,35 \%) \\
6(8,82 \%) \\
7(10,29 \%) \\
3(4,41 \%)\end{array}$ & $\begin{array}{c}0(0 \%) \\
1(1,47 \%) \\
4(5,88 \%) \\
3(4,41 \%)\end{array}$ & 0,0178 \\
\hline $\begin{array}{l}\text { Renda (salários mínimos) } \\
\begin{array}{c}1 \text { a } 2 \\
2 \text { a } 5 \\
>5\end{array}\end{array}$ & $\begin{array}{c}5(7,35 \%) \\
26(38,23 \%) \\
8(11,76 \%)\end{array}$ & $\begin{array}{c}7(10,29 \%) \\
5(7,35 \%) \\
9(13,23 \%)\end{array}$ & $\begin{array}{l}1(1,47 \%) \\
4(5,88 \%) \\
3(4,41 \%)\end{array}$ & 0,0275 \\
\hline $\begin{array}{c}\text { CC }(\mathrm{cm}) \\
\geq 80 \% \\
\geq 88 \% \\
\text { Normal } \circ\end{array}$ & $\begin{array}{c}1(2,38 \%) \\
1(2,38 \%) \\
24(57,14 \%)\end{array}$ & $\begin{array}{c}4(9,52 \%) \\
5(11,90 \%) \\
3(7,14 \%)\end{array}$ & $\begin{array}{c}1(2,38 \%) \\
3(7,14 \%) \\
0(0 \%)\end{array}$ & $<0,0001$ \\
\hline $\begin{array}{l}\text { CC }(\mathrm{cm}) \\
\geq 94 o^{7} \\
\geq 1020^{7} \\
\text { Normal on }\end{array}$ & $\begin{array}{c}0(0 \%) \\
0(0 \%) \\
13(50 \%)\end{array}$ & $\begin{array}{c}4(15,38 \%) \\
0(0 \%) \\
4(15,38 \%)\end{array}$ & $\begin{array}{c}2(7,69 \%) \\
3(11,53 \%) \\
0(0 \%)\end{array}$ & $<0,0001$ \\
\hline
\end{tabular}

$\mathrm{n}=$ número de pessoas; $\mathrm{CC}=$ circunferência da cintura; *Teste $\mathrm{G}$ comparação entre as classificações de IMC, $p$ significante quando $<0,05 ; \%=$ gênero feminino; $o^{*}=$ gênero masculino. 
A obesidade abdominal, classificada pela CC, revelou frequências de risco aumentado em $12(17,65 \%)$ dos pesquisados, e risco substancialmente aumentado, em 12 (17,65\%). Analisando o mesmo parâmetro por gênero, o feminino apresentou risco aumentado em 6 (14,28 \%) mulheres, e substancialmente aumentado, em 9 (21,43 $\%)$; e o masculino mostrou em $6(23,08 \%)$ e 3 $(11,54 \%)$ homens, respectivamente. A normalidade foi observada em 27 (64,29\%) mulheres, e em 17 (65,38\%) homens, não houve diferença significativa entre os sexos.

As prevalências de obesidade e sobrepeso aumentaram com o aumento da CC. Observouse uma forte associação entre IMC e CC, em ambos os sexos, como mostram os valores na Tabela 3.

A presença de hipercolesterolemia em 6 $(8,82 \%)$ voluntários, e hipertrigliceridemia em 5 $(7,35 \%)$, autorreferida apresentaram uma correlação positiva e moderada ( $\mathrm{p}=0,0013 \mathrm{r}=0,3807)$, pois foi uma constante.

Em relação aos hábitos comportamentais, observou-se que a maioria não era tabagista (61 voluntários ou 89,70\%). A atividade física de quase totalidade da amostra foi classificada como praticante de atividade física (44 participantes ou $64,70 \%$ ). Quando se estuda a estratificação por renda, distribuída por faixas encontrou-se um percentual de um a dois $(19,1 \%)$, dois a cinco $(51,5 \%)$ e mais do que cinco salários mínimos $(29,4 \%)$. Em relação ao consumo de bebidas alcoólicas, verificou-se que $33(48,5 \%)$ dos entrevistados apresentaram maior consumo; 21 (30,9\%) consomem nos fins de semana; 13 (19,1\%) não utilizam, e $1(1,5 \%)$ bebe todos os dias.

Em relação ao consumo alimentar dos pesquisados, foi calculado o percentual de consumo dos oito grupos alimentares e classificado como proposto, acima do proposto e abaixo do proposto. Todos esses valores estão descritos na Tabela 4. Foram utilizadas como padrão de comparações as diretrizes que se encontram no Guia Alimentar para população Brasileira.

Tabela 3: Força de correlação entre índice de massa corporal e as variáveis estudadas

\begin{tabular}{c|c|c}
\hline \multirow{2}{*}{ Variáveis } & \multicolumn{2}{|c}{ IMC } \\
\cline { 2 - 3 } & $p$ & $\mathrm{R}$ \\
\hline CC & $0,0001 *$ & 0,8586 \\
\hline HAS & $0,0269 * *$ & 0,2683 \\
\hline Hipertrigliceridemia & $0,0017 * *$ & 0,3737 \\
\hline Ingestão de gordura & $0,0036 * *$ & 0,3478 \\
\hline
\end{tabular}

CC: circunferência da cintura, IMC: índice de massa corporal, HAS: hipertensão arterial sistêmica, * Correlação de Pearson; ** Matriz de correlação.

Tabela 4: Anólise do consumo alimentar dos funcionórios de um serviço público adequada ao número proposto de porções dos grupos alimentares, conforme o Guia alimentar para população brasileira

\begin{tabular}{|c|c|c|c|c|c|c|c|}
\hline \multirow{3}{*}{$\begin{array}{l}\text { Grupo dos } \\
\text { alimentos }\end{array}$} & \multicolumn{7}{|c|}{ Análise de adequação } \\
\hline & \multirow[t]{2}{*}{$\begin{array}{c}\text { Número de porções } \\
\text { adequadas }\end{array}$} & \multicolumn{2}{|c|}{$\begin{array}{l}\text { Conforme o } \\
\text { proposto }\end{array}$} & \multicolumn{2}{|c|}{$\begin{array}{l}\text { Abaixo do pro- } \\
\text { posto }\end{array}$} & \multicolumn{2}{|c|}{ Acima do proposto } \\
\hline & & $\mathrm{n}$ & $\%$ & $\mathrm{n}$ & $\%$ & $\mathrm{n}$ & $\%$ \\
\hline Cereais & 6 & 19 & 27,94 & 40 & 58,82 & 9 & 13,23 \\
\hline Leguminosas & 1 & 10 & 14,7 & 52 & 76,47 & 6 & 8,82 \\
\hline Carnes & 1 & 15 & 22,05 & 9 & 13,23 & 44 & 64,7 \\
\hline Leites & 3 & 6 & 8,82 & 58 & 85,29 & 4 & 5,88 \\
\hline Hortaliças & 3 & 5 & 7,35 & 58 & 85,29 & 5 & 7,35 \\
\hline Frutas & 3 & 5 & 7,35 & 51 & 75 & 12 & 17,64 \\
\hline Açucares & 1 & 14 & 20,58 & 10 & 14,7 & 44 & 64,7 \\
\hline Gorduras & 1 & 20 & 29,41 & 8 & 11,76 & 40 & 58,82 \\
\hline
\end{tabular}

$\mathrm{n}=$ número de pesquisados. 
$\mathrm{Na}$ análise pela descrição da média \pm desvio-padrão $(\mathrm{m} \pm \mathrm{dp})$ do número de porções alimentares, conforme o grupo, em consonância com o Guia Alimentar para população Brasileira, identificaram-se os seguintes valores: $4,94 \pm 1,24$, cereais; $0,34 \pm 0,68$ leguminosas; $1,62 \pm 0,88$, carnes; 1,62 $\pm 1,07$, leites; $1,28 \pm 1,22$, hortaliças; 1,53 $\pm 1,62$ frutas e $1,97 \pm 1,26$, açucares, e 1,72 $\pm 1,02$, gorduras.

Quanto à CC, os pesquisados que referiram maior consumo de doces mostraram fraca relação positiva com DM autorreferido $(r=0,244$ e $p=0,0445)$. Referente à CC e à HAS, não houve relação positiva entre os pesquisados $(r=-0,1951$; $p=0,1108$ ).

\section{Discussão}

A adiposidade central, indicando maior distribuição de tecido adiposo abdominal, está associada com sobrepeso e obesidade nos participantes. Assim como em outros estudos, foi observada uma alta correlação entre CC e IMC ${ }^{2}$, 18, 19, 12 , sendo amplamente conhecido que a distribuição central de adiposidade está associada a distúrbios metabólicos e risco cardiovascular ${ }^{20}$.

Neste estudo, ressaltamos a associação do aumento da adiposidade corporal na prevalência de hipertensão arterial. Os resultados de IMC e da HAS se associam nos voluntários em ambos os sexos. Outros estudos encontraram a mesma associação. Por outro lado, não houve relação entre a CC e a HAS nos pesquisados, diferentemente de outros estudos em que houve forte associação ${ }^{6,21,22,23}$. Este resultado não mostrou um número expressivo de pessoas com HAS, talvez por não ter sido utilizado o método de aferição da pressão arterial para diagnosticar o número real de hipertensos entre os participantes.

Os dados apresentados sobre o estado nutricional mostraram que a obesidade foi mais frequente no sexo masculino $(19,23 \%)$ do que no feminino $(9,52 \%)$, porém o sobrepeso não teve diferença significativa entre as mulheres $(30,95 \%)$ e os homens (30,76\%). Nesse sentido, a prevalên- cia de obesidade encontrada foi mais elevada do que a verificada pela Pesquisa do Orçamento Familiar (POF) 2002-2003 para os homens $(6,7 \%)$ e menor para as mulheres $(11,7 \%)$ do Nordeste brasileiro $^{24}$. Em outro estudo realizado na capital do Estado da Bahia, em 2001, a prevalência de obesidade identificada entre mulheres $(15,1 \%)$ e homens $(8,4 \%)$ mostrou que a obesidade encontrada nos homens é menor se comparada com os pesquisados aqui, e maior, para as mulheres participantes também desta pesquisa ${ }^{12}$.

Em relação ao questionário de frequência alimentar, observou-se uma diminuição no consumo de cereais e uma elevação no de açucares, de carnes e de gorduras. Enquanto, a ingestão das hortaliças, frutas, produtos lácteos e leguminosas mantiveram-se abaixo do proposto. Resultados semelhantes foram encontrados na dieta da população nas regiões metropolitanas, em que foram observados um excessivo consumo de açúcar e de gorduras totais e saturadas, assim como insuficiente ingestão de frutas e hortaliças ${ }^{25}$. Entretanto, São Paulo diferenciouse apenas no maior consumo de leguminosas, com apenas $37,1 \%$ abaixo do proposto, e no grupo das gorduras, com somente $36,7 \%$ acima do proposto $^{26}$.

Dados nacionais, no período de 1975 a 2003, apontaram que alimentos tradicionais da dieta do brasileiro, como o arroz e o feijão, perderam a importância. Em contrapartida, o consumo de produtos industrializados, como biscoitos e refrigerantes, aumentou em $400 \%{ }^{25}$. Resultados semelhantes foram encontrados num estudo realizado na Espanha, em que alimentos, como carboidratos complexos ricos em fibras, hortaliças e frutas, estão sendo substituídos por outros de maior densidade energética, ricos em gordura e açucares ${ }^{8}$. Marcondelli et al..$^{27}$ encontraram elevado percentual de inadequação em relação à alimentação em seu estudo, com um menor consumo de leite e derivados $(23,1 \%)$, frutas e vegetais $(24,9 \%)$ e carboidratos complexos $(25,9 \%)$. Destaca-se também o alto consumo de refrigerante e doce $(74,0 \%)$. Esses achados, segundo os autores, também foram encontrados na POF 
$(2002 / 2003)^{24}$, que mostra a média de frutas e verduras consumidas pela população brasileira muito abaixo das recomendações.

O baixo consumo de frutas e vegetais mostrou-se generalizado nas 14 regiões geográficas do planeta, abrangendo Europa, Mediterrâneo Oriental, África, América, Ásia e Pacífico Ocidental, estudadas em amplo projeto desenvolvido pela OMS. Tomando-se como referência $600 \mathrm{~g}$ de fruta e/ou verduras/dia, a média de consumo na totalidade das regiões esteve abaixo dessa recomendação ${ }^{28}$. Esses resultados alertam para o preocupante aumento da procura por alimentos ricos em açúcares e diminuição da preferência pelas frutas, legumes e verduras, que tem ocorrido no Brasil, nos últimos anos ${ }^{29}$.

$\mathrm{O}$ alto consumo de açúcar e gorduras e a ingestão insuficiente de frutas e hortaliças pela população mundial, segundo os dados apresentados, apontam a necessidade de estabelecer estratégias para redução do consumo de alimentos com alta densidade energética e, ao mesmo tempo, aumentar a ingestão daqueles que são reconhecidamente fatores de proteção para a saúde. É preciso assegurar a manutenção do consumo de leguminosas, tubérculos e raízes, fontes importantes e fundamentais de carboidratos, bem como garantir que sejam consumidos produtos lácteos, principais fontes de cálcio na alimentação humana, além de fonte de proteínas de alto valor biológico ${ }^{11}$. As frutas e verduras são alimentos fundamentais para prevenção de patologias, como as doenças cardiovasculares e certos tipos de câncer, principalmente do trato digestivo ${ }^{3}$.

\section{Conclusão}

O aumento do excesso de peso e a alimentação inadequada da população, observados na literatura, comprovam os resultados encontrados neste estudo. $\mathrm{O}$ que indica a necessidade de ações que visem à saúde, ou seja, voltadas à prevenção e ao controle de doenças, de modo a contribuir para a redução de enfermidades crô- nicas relacionadas ao excesso de peso e, concomitantemente, a uma alimentação inadequada.

Os Guias Alimentares, como o Guia Alimentar para a população Brasileira, estabelecem condutas com o objetivo principal de orientar a população, em geral, a ter uma alimentação mais adequada, por esse motivo eles favorecem a adesão a hábitos alimentares saudáveis, contribuindo, assim, para a manutenção de um peso adequado.

\section{Referências}

1. Peña M, Bacallao J. La obesidad y sus tendências en la región. Rev Panam Salud Pública. 2001;10(2):75-8.

2. Sousa RMRP, Sobral DP, Paz SMRS, Martins MCC. Prevalência de sobrepeso e obesidade entre funcionários plantonistas de unidades de saúde de Teresina, Piauí. Rev Nutr Campinas. 2007;20(5):47382.

3. Organização Pan-Americana de saúde. Organização Mundial de saúde. Doenças crônicas degenerativas e obesidade: estratégia mundial sobre alimentação saudável, atividade fisica e saúde. Brasília, DF. 2003; p. 29.

4. Francischi RPP, Pereira LO, Freitas CS, Klopfer M, Santos RC, Vieira P, et al. Obesidade: atualização sobre etiologia, morbidade e tratamento. Rev Nutr. $2000 ; 13: 17-28$.

5. Lerario DDG, Gimeno SG, Franco LJ, Iunes M, Ferreira RG. Excesso de peso e gordura abdominal para a síndrome metabólica em nipo-brasileiros. Rev Saúde Pública. 2002;36:4-11.

6. Mariath AB, Grillo LP, Silva RO, Schmitz P, Campos IC, Medina JRP, Kruger R M. Obesidade e fatores de risco para o desenvolvimento de doenças crônicas não transmissíveis entre usuários de unidade de alimentação e nutrição. Cad Saúde Pública. 2007;23(4):897-905.

7. Monteiro CA, Moura EC, Jaime PC, Lucca A, Florindo AA, Figueiredo ICR, et al. Monitoramento de fatores de risco para doenças crônicas por entrevistas telefônicas. Rev Saúde Pública. 2005;39:47-57.

8. Arnaiz MG. La emergência de las sociedades obesogénicas o de la obesidad como problema social. Rev Nutr Campinas. 2009;22(1):5-18. 
9. Romanzini M, Felipe FR, Lopes AS, Petroski EL, Farias Júnior JC. Prevalência de fatores de risco cardiovascular em adolescentes. Cad Saúde Pública. 2008;24(11):2573-81.

10. Kain J, Vio F, Albala C. Obesity trends and determinant factors in Latin América. Cad Saúde Pública. 2003;19 Suppl 1):S77-S86.

11. Ministério da Saúde (Brasil), Secretaria de atenção a saúde, Coordenação Geral da Política de Alimentação e Nutrição, Guia Alimentar para a População Brasileira: promovendo a alimentação saudável, Brasília: Ministério da Saúde, 2006.

12. Oliveira LPM, Assis AMO, Silva MCM, Santana MLP, Santos NS, Pinheiro SMC, Barreto ML, Souza CO. Fatores associados a excesso de peso e concentração de gordura abdominal em adultos na cidade de Salvador, Bahia, Brasil. Cad Saúde Pública. 2009;25(3):570-82.

13. Martins IS, Marinho SP. O potencial diagnóstico dos indicadores da obesidade centralizada. Rev Saúde Pública. 2003;37:760-7.

14. Ministério da Saúde (Brasil), Universidade Federal de Goiás, Centro Colaborador em Alimentação e Nutrição da Região Centro-Oeste. Antropometria: manual de técnicas e procedimentos. Vigilância nutricional. Goiânia: Ministério da Saúde. 2. ed., 2003.

15. World Health Organization. Obesity. What are health consequences of being overweight? 2000: 1-11. [acesso em: 2009 nov 15]. Disponível em: <http:// www.who.int/features/qa/49/en/index.html>.

16. Ribeiro AC, Sávio KEO, Rodrigues MLCF, Costa THM, Schmitz BAS. Validação de um questionário de freqüência de consumo alimentar para população adulta. Rev Nutr Campinas. 2006;19(5):553-62.

17. Ayres M. Bioestat. Versão 5.0. Soc. Civ. Mamirauá. 2007. MCT - CNPq. Belém.

18. Peixoto MRG, Benício MH D'A, Latorre MRDO, Jardim PCBV. Circunferência da cintura e índice de massa corporal como preditores da hipertensão arterial. Arq Bras Cardiol. 2006;87:462-70.

19. Sampaio LR, Figueiredo VC. Correlação entre o índice de massa corporal e os indicadores antropométricos de distribuição de gordura corporal em adultos e idosos. Rev Nutr Campinas. 2005;18-61.
20. Oliveira CL, Mello MT, Cintra IP, Fisberg M. Obesidade e síndrome metabólica na infância e adolescência. Rev Nutr Campinas. 2004; 17(2):237-245.

21. Sarno F, Monteiro CA. Importância relativa do Índice de Massa Corporal e da circunferência abdominal na predição da hipertensão arterial. Rev Saúde Pública. 2007;41(5):788-96.

22. Jardim PCBV, Gondim MRP, Monego ET, Moreira HG, Vitorino PVO, Souza WKSB, et al. Hipertensão arterial e alguns fatores de risco em uma capital brasileira. Arq Bras Cardiol. 2007;88(4):452-7.

23. Carneiro G, Faria AN, Ribeiro Filho FF, Guimarães A, Lerário D, Ferreira SRG, et al. Influência da distribuição da gordura corporal sobre a prevalência de hipertensão arterial e outros fatores de risco cardiovascular em indivíduos obesos. Rev Assoc Med Bras. 2003;49(3):306-11.

24. Instituto Brasileiro de Geografia e Estatística. Pesquisa de orçamento familiares 2002-2003. Análise da disponibilidade domiciliar de alimentos e do estado nutricional no Brasil. Rio de Janeiro: Instituto Brasileiro de Geografia e Estatística; 2006.

25. Neumann AICP, Shirassu MM, Fisberg RM. Consumo de alimentos de risco e proteção para doenças cardiovasculares entre funcionários públicos. Rev Nutr Campinas. 2006;19(1):19-28.

26. Fiore EG, Araújo M, Campos VJ, Mattos LL, Pelliciari MC, Nuzzo L. Perfil de indivíduos com diferentes níveis de sobrepeso e avaliação do consumo alimentar frente às diretrizes do guia alimentar para a população brasileira. Rev Nutr. 2007;11-15.

27. Marcondelli P, Costa THM, Schmitz BAS. Nível de atividade física e hábitos alimentares de universitários do $3^{\circ}$ ao $5^{\circ}$ semestres da área da saúde. Rev Nutr Campinas. 2008;21(1):39-47.

28. Lock K, Pomerlau J, Causer L, Altmann DR, McKee M. The global burden attributable to low consumption of fruit and vegetables: implications for global strategy on diet. Bull World Health Organ. 2005;83(2):100-8.

29. Levy-Costa RB, Sichieri R, Pontes NS, Monteiro CA. Disponibilidade domiciliar de alimentos no Brasil: distribuição e evolução (1974-2003). Rev Saúde Publica. 2005; 39(4): 530-40. 